\title{
A case report of moyamoya disease in children treated with encephalo-duro-myo- arterio-pericranial synangiosis
}

Julius July

pISSN: 0853-1773 - elSSN: 2252-8083 https://doi.org/10.13181/mji.cr.204452 Med J Indones. 2021;30:228-31

Received: December 30, 2019

Accepted: September 03, 2020

Published online: May 11, 2021

Author's affiliations:

Department of Neurosurgery, Faculty of Medicine, Universitas Pelita Harapan, Tangerang, Indonesia; Neuroscience Center, Siloam Hospital, Tangerang, Indonesia

\section{Corresponding author:}

Julius July

Department of Neurosurgery, Faculty of Medicine, Universitas Pelita Harapan, Neuroscience Center, Siloam Hospital, Jalan Siloam No. 6, Lippo Village,

Tangerang 15811, Indonesia

Tel/Fax: +62-21-5460055 ext. 5731/

+62-21-5460921

E-mail: juliusjuly@yahoo.com

\begin{abstract}
Moyamoya disease that manifests during childhood may pose a special challenge for surgeons. We report a case of a 10-year-old girl who suffered from moyamoya disease and was successfully treated with encephalo-duro-myo-arterio-pericranial synangiosis (EDMAPS). She presented with a recurrent transient ischemic attack that worsened for 1 year. She was aphasic globally (sensory and motor) and had slightly weak right extremities. Her magnetic resonance angiography and computed tomography angiography showed the typical features of moyamoya disease with bilateral stenosis at the terminal internal carotid artery, bilaterally abnormal vascular networks, and a left ischemic event involving the temporoparietal region. She was recovered well after underwent bilateral EDMAPS, fully regained her language function after 3 months, and gathered her strength back. Therefore, EDMAPS could be a good, safe, and effective treatment for moyamoya disease in children.
\end{abstract}

KEYWORDS encephalo-duro-myo-arterio-pericranial synangiosis, moyamoya disease, stroke, surgery, transient ischemic attack
Moyamoya disease is a disease characterized by the narrowing of the terminal internal carotid artery (ICA) or the proximal areas of the middle and anterior cerebral arteries and compensated with abnormal vascular networks near stenotic lesions. ${ }^{1}$ The incidence of moyamoya disease is higher in East Asia, especially Japan and South Korea, than in other regions, and it predominantly affects females. It has two peaks of onset age: between 5 and 9 years and between 45 and 49 years. ${ }^{1}$

Children with cerebrovascular event (stroke) should always be cautious of moyamoya disease. They usually present with middle cerebral artery (MCA) territory stroke, such as hemiparesis, aphasia, hemihypesthesia and visual field deficit. Computed tomography (CT) angiography investigation is used to confirm the diagnosis and also to rule out other differential diagnosis, such as cerebral vasculitis (tuberculosis, Kawasaki disease, or other causes) and some metabolic disorders. Moyamoya disease that manifests during childhood is a special case because of its small vessel caliber. ${ }^{2}$

Moyamoya disease can be managed by surgical revascularization through direct bypass (anastomosis), indirect revascularization, and a combination of both techniques. ${ }^{2}$ Most of the previous indirect bypass procedures involved MCA territory. Thus, encephaloduro-myo-arterio-pericranial synangiosis (EDMAPS) revascularization was developed to revascularize the anterior cerebral artery territory using the frontal 
pericranial flap through medial frontal craniotomy and additional indirect revascularization with an insertion of reversed dural pedicles. ${ }^{1}$ This report showed a case of successful treatment of EDMAPS revascularization for moyamoya disease in children.

\section{CASE REPORT}

A 10-year-old girl was admitted because of headache, aphasia, and weakness of the right upper extremity followed by the lower extremity for 25 days. She also had a history of multiple transient ischemic attacks (TIAs) for the last 3 years, and it worsened since a year ago. She never had any seizure. During the examination, she was globally aphasic, but she expressed that she was having a headache through nonverbal language. Her brain magnetic resonance imaging $(\mathrm{MRI})$ revealed multiple watershed zone infarcts on the left temporoparietal side (Figure 1). The CT angiography showed a "puff of smoke" image as a sign of moyamoya collaterals in the basal ganglia region and severe stenosis of the bilateral terminal ICA (Figure 2).
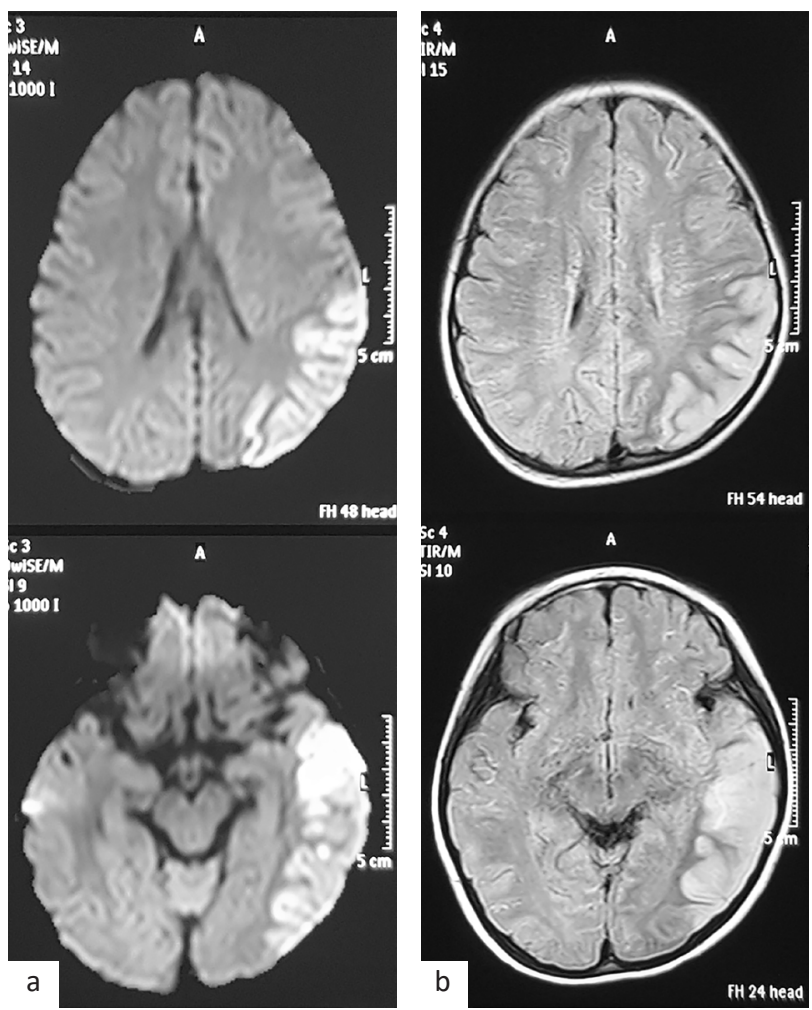

Figure 1. MRI examination of moyamoya disease. (a) Diffusionweighted imaging (DWI) sequence and (b) flair sequence of the brain magnetic resonance imaging (MRI) shows an infarct on the left temporoparietal side (middle cerebral artery [MCA] distribution)
A surgery was planned 1 month after the initial ischemic event. The first EDMAPS revascularization was performed on the left side. The patient must be hydrated prior to surgery, and this step must be included in preoperative management. She was placed in a supine position, and her head was slightly turned to the contralateral side. The skin was incised following the parietal branch of the superficial temporal artery (STA), extended upward until the midline at the bregma, and curved downward to the hairline. The parietal branch of the STA that crossed the skin incision was dissected away from the surrounding tissues under a microscope, and the artery was small (less than $1 \mathrm{~mm}$ ). The temporal muscle should be dissected widely and made as a vascularized flap for encephalomyosynangiosis (EMS) while avoiding too much coagulation (Figure $3 a$ ). In the next step, a vascularized frontal pericranium consisting of the periosteum with an overlying loose areolar layer was prepared for encephalo-pericranial synangiosis. Dissection was carefully conducted to preserve the vascular pedicle of the pericranial flap. After the bone was exposed, indocyanine green was applied to mark the middle meningeal artery (MMA) before frontotemporal craniotomy was performed (Figure 3b). In craniotomy, the MMA was avoided, and the sphenoid wing was removed carefully using a rongeur and a high-speed drill (Figure 3, c and d). Next, the dura was opened in the form of a stellate, while the main branch of the MMA was preserved (Figure 3e). Dural pedicles were reversed and inserted into the epiarachnoid space, and the brain surface was covered with the frontal pericranial flap and the temporal muscle (Figure $3 f$ ). Cranioplasty was performed by utilizing the temporal fascia that mimicked the temporal muscle to
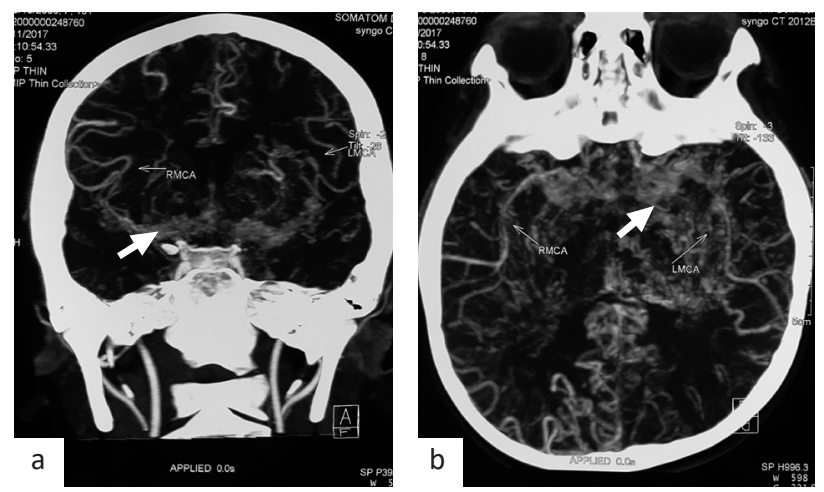

Figure 2. Computed tomography (CT) angiography of moyamoya disease patient. (a) the terminal internal carotid artery (ICA) stenosis (arrow) with basal moyamoya collaterals and (b) a typical "puff of smoke" image (stage 3) (arrow) 

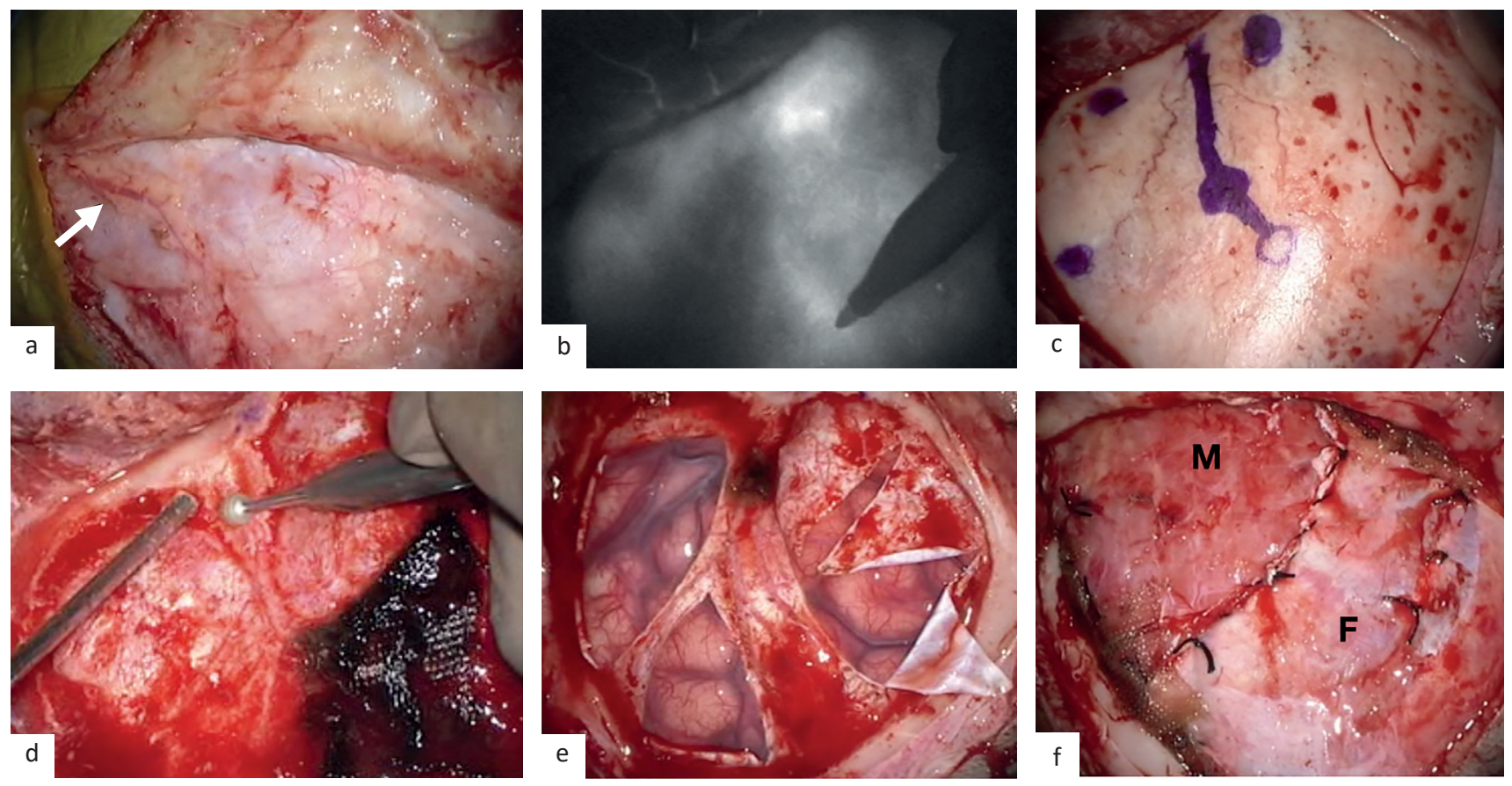

Figure 3. Intraoperative photographs of EDMAPS. (a) The diameter of the STA was less than $1 \mathrm{~mm}$ (arrow); (b) ICG was used to visualize the MMA before a burr hole was created; (c) the location of burr holes and the MMA track were evident; (d) the sphenoid ridge was drilled to preserve the MMA; (e) the dura was opened in a stellate form, reversed, and inserted into the epiarachnoid space while preserving the main branches of the MMA; $(f)$ the brain surface was covered with the frontal pericranial flap (letter F) and the temporal muscle (letter M). EDMAPS=encephalo-duro-myo-arterio-pericranial synangiosis; ICG=indocyanine green; $M M A=$ middle meningeal artery; STA=superficial temporal artery

cover the inserted bone dust to avoid head deformity. The incision was closed layer by layer, and the total operation time was about 3 hours with no blood transfusion needed. During surgery, $\mathrm{PaCO}_{2}$ should be around $40 \mathrm{mmHg}$. She was given an antiplatelet 3 days after the surgery.

After the surgery, the patient was hospitalized for five more days and discharged with mild weakness on the right side. After 1 month of follow-up, the patient had a second EDMAPS for the right brain. She recovered well after the second surgery, fully regained her language function after 3 months, and gathered her strength back. One-year follow-up after surgery showed no neurological deficits.

\section{DISCUSSION}

Children with moyamoya disease present with TIA or cerebral infarction. Brain ischemia or infarction can cause intellectual impairment and sensory and motor dysfunctions. This patient had headaches and verbal problems. Hence, head CT scan was initially done to observe area of infarction, any hemorrhage, or any atrophy. The MRI combined with magnetic resonance angiography shows sensitivity of $92 \%$ and specificity of $100 \%$ in diagnosing moyamoya disease.,34 Meanwhile, altered mental status in children has vast differential diagnosis. It can be caused by extracranial causes like toxin, infection, and metabolic disease and intracranial causes like structural brain problem or vascular abnormality. ${ }^{5}$ In this case, sign of increased intracranial pressure and central nervous system infection was ruled out with initial head CT scan show brain infarction. The diagnosis of moyamoya disease was confirmed by MRI or CT angiography.

In this case, it was impossible to perform direct bypass due to small vessel of the donor and recipient. Direct bypass is an anastomosis procedure between two arteries, i.e., from the STA to the MCA or from the occipital artery to the posterior cerebral artery. This procedure can consequently increase blood flow directly, but it is highly dependent on the experience of surgeons and the diameter of the arteries of recipients and donors. Therefore, direct anastomosis was difficult to be performed in patients with small diameter of recipient and donor artery, especially pediatric patients and patients with advanced moyamoya disease. This procedure may also cause 
hyperperfusion syndrome, resulted to functional deterioration and intracerebral hemorrhage. ${ }^{6}$

Indirect revascularization was performed in this patient; nevertheless, it had a high chance of collateral formation because of the pediatric age. Indirect revascularization is a procedure to promote spontaneous leptomeningeal collateral connection from various connective tissues. It can be performed through several methods, such as EMS, encephaloduroarteriosynangiosis, encephalo-duroarterio-myo-synangiosis, encephalo-galeo-synangiosis, dural inversion, multiple burr hole surgery, ${ }^{1}$ or EDMAPS. Indirect revascularization is easier, but do not provide immediate effects compared to direct bypass because surgical collaterals need 3-4 months to develop. Therefore, patients still have a risk of suffering from an ischemic event post-operation until collateral arteries develop. The effectiveness of indirect bypass is around $50-60 \%$ in adults and more than $60 \%$ in pediatric patients with moyamoya disease. Therefore, a combined bypass has advantages of both procedures and is widely used for moyamoya disease management. ${ }^{7,8}$

EDMAPS was chosen as the indirect bypass procedure in this case. Kuroda et $a^{9}$ prospectively studied 75 patients ( 28 pediatrics with 47 procedures and 47 adults with 76 procedures) who underwent STA-MCA anastomosis and EDMAPS for moyamoya disease management. The 3-month mortality and morbidity rates of pediatric patients were $0 \%$ and $4.3 \%$, respectively; in adult patients, the corresponding rates were $0 \%$ and $6.6 \%$. In pediatric patients, no cerebrovascular events occurred during the mean follow-up period of 72.8 months. However, one adult patient developed cerebral infarction on the right occipital lobe because of progressive occlusion at the right posterior cerebral artery about 11 months after surgery. The yearly risk of cerebrovascular events in adults was about $0.4 \%$. This study showed that STAMCA anastomosis with EDMAPS is safe and effective for improving the long-term outcome of moyamoya disease. ${ }^{9}$ Another study demonstrated that indirect revascularization with EDMAPS is effective in $95 \%$ of pediatric cases. ${ }^{6}$

During EDMAPS, MMA preservation is important because it can supply a collateral connection from the dura mater. Although the MMA was successfully preserved, it can easily be injured during standard frontotemporal craniotomy. A diamond drill was used because the MMA was a monorail type. Many studies on the anatomy of MMA have been performed, whereas only one study analyzed the surgical point of view between the MMA and the sphenoid bone to develop an enhanced technique in preserving the MMA during the procedure. Hori et al ${ }^{10}$ classified the MMA into three types and suggested the methods to preserved the MMA, e.g.: (1) in bridge type (shallow groove), it was suggested to remove the sphenoid bone using a rongeur; (2) in monorail type (deep groove), it was suggested to use a high-speed diamond drill to remove the lesser wing; (3) in tunnel type (bony tunnel), a high-speed drill is also suggested, although it is difficult because the MMA can be easily injured..$^{10}$

Patient with periodic $\mathrm{CT}$ angiography and perfusion requires follow-up every few years to ensure an adequate brain perfusion. This case report shows that EDMAPS may be a good, safe, and effective treatment option for moyamoya disease in children.

Conflict of Interest

The author affirm no conflict of interest in this study.

\section{Acknowledgment \\ None. \\ Funding Sources \\ None.}

\section{REFERENCES}

1. Kuroda S, Houkin K. Moyamoya disease: current concepts and future perspectives. Lancet Neurol. 2008;7(11):1056-66.

2. Scott RM, Smith ER. Moyamoya disease and moyamoya syndrome. N Engl J Med. 2009;360(12):1226-37.

3. Yamada I, Suzuki S, Matsushima Y. Moyamoya disease: comparison of assessment with MR angiography and MR imaging versus conventional angiography. Radiology. 1995;196(1):211-8.

4. Katz DA, Marks MP, Napel SA, Bracci PM, Roberts SL. Circle of Willis: evaluation with spiral CT angiography, MR angiography, and conventional angiography. Radiology. 1995;195(2):445-9.

5. Nagiub M, Allarakhia I. Pediatric Moyamoya disease. Am J Case Rep. 2013;14:134-8.

6. Kim T, Oh CW, Bang JS, Kim JE, Cho WS. Moyamoya disease: treatment and outcomes. J Stroke. 2016;18(1):21-30.

7. Uchino H, Kim JH, Fujima N, Kazumata K, Ito M, Nakayama N, et al. Synergistic interaction between direct and indirect bypasses in combined procedures: the significance of indirect bypasses in moyamoya disease. Neurosurgery. 2016;80(2):201-9.

8. Amin-Hanjani S, Singh A, Rifai H, Thulborn KR, Alaraj A, Aletich $\checkmark$, et al. Combined direct and indirect bypass for moyamoya: quantitative assesment of direct bypass flow over time. Neurosurgery. 2013;73(6):962-7.

9. Kuroda S, Houkin K, Ishikawa T, Nakayama N, Iwasaki Y. Novel bypass surgery for moyamoya disease using pericranial flap: its impacts on cerebral hemodynamics and long-term outcome. Neurosurgery. 2010;66(6):1093-101.

10. Hori S, Kashiwazaki D, Akioka N, Tomohide H, Hori E, Umemura $K$, et al. Surgical anatomy and preservation of the middle meningeal artery during bypass surgery for moyamoya disease. Acta Neurochir. 2015;157(1):29-36. 